\title{
Deep Learning-based Computer Vision for Radiation Defect Analysis: from Static Defect Segmentation to Dynamic Defect Tracking
}

Rajat Sainju ${ }^{1}$, Wei-Ying Chen ${ }^{2}$, Samuel Schaefer ${ }^{3}$, Graham Roberts ${ }^{3}$, Mychailo Toloczko ${ }^{4}$, Danny Edwards ${ }^{4}$, Meimei $\mathrm{Li}^{2}$ and Yuanyuan $\mathrm{Zhu}^{5}$

${ }^{1}$ University of Connecticut, United States, ${ }^{2}$ ANL, United States, ${ }^{3}$ UConn, United States, ${ }^{4}$ PNNL, United States, ${ }^{5}$ University of Connecticut, Storrs, Connecticut, United States

Over the past several decades, immense research has been dedicated to the understanding of radiation effects on materials, particularly driven by the needs of the nuclear power industry. Yet, researchers have been unable to reach a unified and comprehensive predictive insight to the response of reactor structural materials under energetic particle irradiation. This is in part due to the lack of satisfactory TEM image processing tools capable of performing reliable, fast, and reproducible radiation defect detection and tracking, leaving experimental TEM data, especially new in situ irradiation TEM video data, underexplored.

Deep learning-based computer vision techniques have undergone a renaissance over the past decade, enabling disruptive advances in applications ranging from sensing for autonomous vehicles to pattern detection for cancer diagnosis, and these advances have only recently begun to be incorporated to the analysis of radiation defects. Here, we show two studies that utilize deep learning-based computer vision methods for semantic segmentation of static radiation-induced (extended) defects, and for automated tracking of the dynamic evolution of defect clusters as irradiation occurs. In the first study, which involves ex situ diffraction contrast STEM [1] images of a neutron-irradiated HT-9 martensitic steel, we developed and trained a novel deep learning architecture, called the DefectSegNet [2] for fast semantic segmentation of extended defects. As shown in Figure 1, this computer vision model DefectSegNet and associated MATLAB algorithms performed defect quantification in a more reproducible and reliable manner in just a few seconds. Additionally, advanced in situ TEM equipped with high-energy ion sources are beginning to produce an 'avalanche' of big data, e.g. gigabytes of in situ TEM videos produced in a single irradiation experiment, making it increasingly difficult to extract and quantify the temporal information of radiation defect dynamics. One of the main challenges in the integration of deep learning with in situ TEM video analysis of radiation defects is a lack of professionally annotated in situ videos of radiation defects, which is required for machine learning training. In the second study, we started with establishing an annotation pipeline for the execution of annotation tasks from fetching raw TEM video frames to preprocessing (e.g. background subtraction, full variance normalization, and drift correction) to creating ground truth labeling through manual annotation. Figure 2 (left) presents an example of ground truth labeling (one out of 380 frames analyzed) of irradiation-induced defect clusters (dislocation loops and stacking-fault tetrahedra) in a pure Nickel irradiated with $1 \mathrm{MeV} \mathrm{Kr}$ ions at $700{ }^{\circ} \mathrm{C}$ and (right) digital processing results of the lifetime distribution of the defect clusters during irradiation. We found that the lifetime of these cascade defect clusters showed a monotonic decay within the first $3800 \mathrm{~ms}$. A considerably higher temporal resolution (10 ms) has been achieved through in situ imaging and digital analysis, which is over two orders of magnitudes higher than that in a previous in situ study of Ni under heavy ion irradiation (5 s) [3]. Such quantitative analysis of the dynamic behavior of radiation defects at the high temporal resolution, after augmentation by a dedicated multiple object tracking (MOT) computer vision model that enables tracking of individual defect clusters over time, holds the promise of advancing our understanding of radiation defect development under irradiation at elevated temperatures. 
We acknowledge funding from the U.S. DOE Office of Fusion Energy Sciences under contract DE-AC0576RL01830, Office of Nuclear Energy's NEET program project CFA 16-10570, and UConn HPC computing facility. Argonne National Laboratory's contribution is based upon work supported by Laboratory Directed Research and Development (LDRD) funding, provided by the Director, Office of Science, of the U.S. Department of Energy under Contract No. DE-AC02-06CH11357.
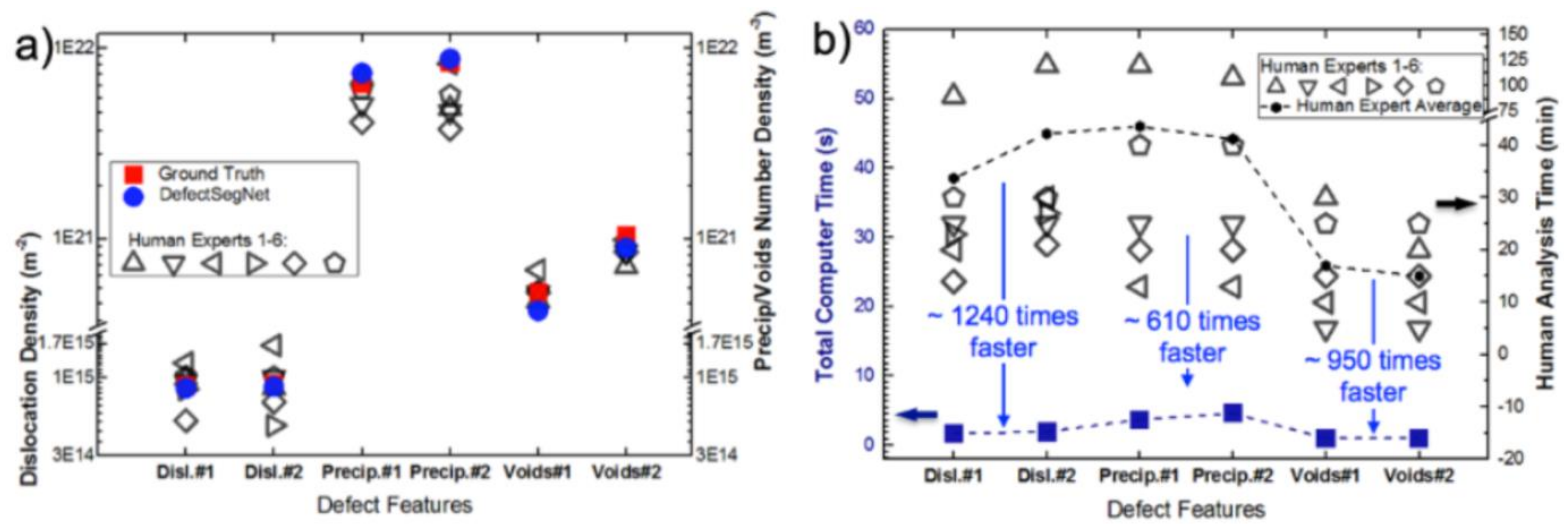

Figure 1. Comparison of static radiation defect quantification performed by computer (DefectSegNet + DefectQuant algorithms) and by human experts. The computer-based method outperformed human experts at a) accuracy and b) efficiency.
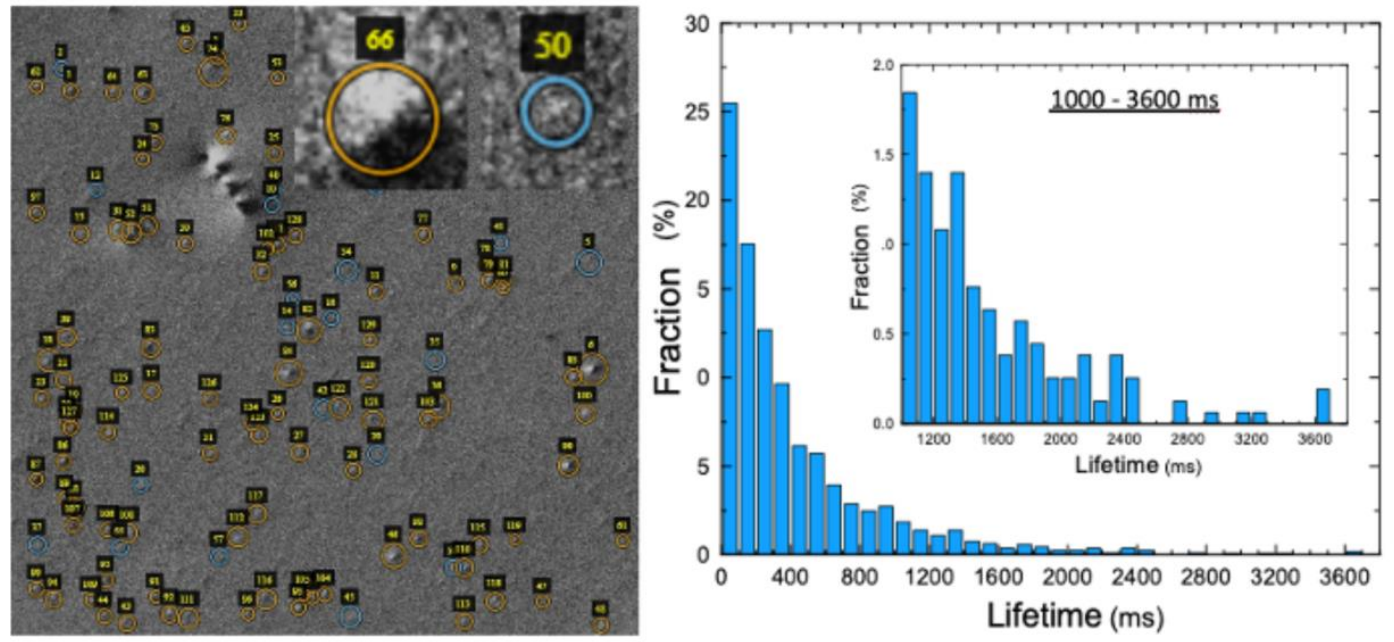

Figure 2. Ground truth annotation and digital processing outcome of the lifetime distribution of dynamic defect clusters (orange: cluster with obvious black-white contrast; blue: no clear contrast interface) in a pure Nickel irradiated with $1 \mathrm{MeV} \mathrm{Kr}$ ions at $700 \circ \mathrm{C}$. Note that both of the recording temporal resolution and the analysis temporal resolution are high $(10 \mathrm{~ms} /$ frame $)$.

\section{References}

[1] Y Zhu, C Ophus, MB Toloczko and DJ Edwards, Ultramicroscopy, 193(2018) 12-23.

[2] G Roberts, S Y Haile, R Sainju, D J Edwards, B Hutchinson and Y Zhu, Scientific Reports 9(2019), 12744

[3] H Sakaida, N Sekimura and S Ishino, Journal of Nuclear Materials, 179(1991) 928-930 